\title{
ON SPECTRUM CALIBRATION FOR NEARSHORE WAVE TRANSFORMATION
}

\author{
Zhong Peng ${ }^{1}$ and Jill Bradon ${ }^{1}$
}

\begin{abstract}
A 'shape-focus' method is proposed for wave spectrum calibration. In this method calibration factors at each frequency bin are derived from measured and modelled data using a least square error approach. Modelled wave spectra are calibrated using the shape-focus method and then applied to a SWAN wave model to simulate nearshore wave transformation. Nearshore wave spectra are then compared with measurements. Results show that spectrum calibration with the shape-focus method improves wave data accuracy significantly for both significant wave height and mean wave periods, thus provides a more accurate solution to calibrating wave spectra for nearshore wave modelling.
\end{abstract}

Keywords: wave spectrum, calibration, SWAN, spectrum shape, nearshore wave, ensemble

\section{INTRODUCTION}

The varying bathymetry and relatively shallow water in nearshore regions lead to complex wave processes and hydrodynamics due to bottom friction, wave refraction, wave diffraction and wave breaking. Therefore, a fine-gridding approach to transform offshore waves is necessary for coastal engineering. The accuracy of offshore wave conditions at model domain boundaries is thus of key importance to the accuracy of the environmental characteristics of the area.

A 2-D wave spectrum represents the offshore wave energy by describing the spatial distribution of wave energy in frequency and direction domains. However, 2-D wave spectra at offshore locations mainly come from global or regional wave hindcast models, and often deviate from the true energy, mainly because of the approximate wind inputs, model resolution, numerical schemes and empirical source terms. There is little research on how to calibrate the offshore wave spectrum. Engineering practice is to scale up the spectrum by applying a constant factor, derived from comparisons of modelled and measured integrated wave parameters, and choosing to either keep wave frequencies the same, or to squeeze frequencies to conserve wave steepness (e.g. Tucker, 1991). However, those methodologies ignore the spectrum evolution, and assume a fixed spectrum shape during calibration, which can result in incorrect energy distribution in frequency domain, and errors in wave energy refraction, mean wave periods and wave spreading. Figure 1 shows the ensembled temporal-varying wave spectrum for both measurements and wave model data at the same location in the east coast of USA. It is clearly shown that the $99^{\text {th }}$-percentile of measured wave spectral displays double peaks, while that of modelled wave spectral presents a dominant swell peak. Therefore, the modelled wave spectrum could not be calibrated simply by scaling magnitudes of spectrum density, as the wave systems are different between model data and measurements.

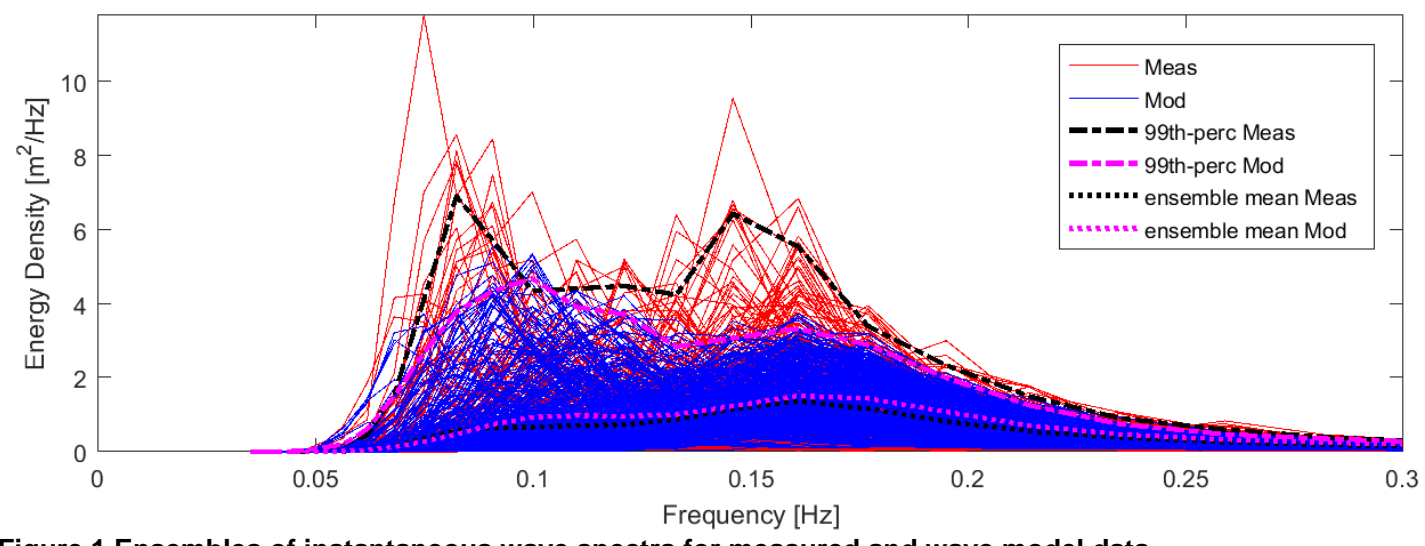

Figure 1 Ensembles of instantaneous wave spectra for measured and wave model data

Instantaneous wave data are the result of a complex balance of wave generation, dissipation and nonlinear interaction. When a wave propagates from the deep ocean to the coast, the main physical

\footnotetext{
${ }^{1}$ Fugro GB Marine Ltd., Fugro House, Hithercroft Road, Wallingford, OX10 9RB, UK
} 
processes change. In the deep ocean, where water depths are large enough, the wind-generated wave grows due to the resonance and feed-back mechanism. As wind velocity and fetch increase, the amount of energy transferred to the waves increases exponentially and wave length increases. In the meantime, quadruplet wave-wave interactions dominate the evolution of the spectrum offshore, and they transfer wave energy from the spectral peak to lower frequencies. These processes lead to a narrow band spectrum. During those processes, wave energy is dissipated or reduced slightly by angular and longitudinal dispersions or whitecapping, which help transfer wave energy from the spectral peak to higher frequencies. When a wave approaches the coast, the influence of the sea bottom on waves becomes significant, through wave refraction, wave shoaling and wave breaking. Furthermore, quadruplet wave-wave interaction gets weak, but triad wave-wave interaction becomes important. Triad wave-wave interaction generates higher harmonics and lower frequencies, resulting in a broader spectrum. This can be observed from Fig.3 in Ardhuin et al. (2007), which presents an example of measured wave spectrum evolution from offshore to the coast. This evolution indicates that the wave spectrum shape is not fixed but keeps varying. Therefore, it is important that the wave spectrum shape should be considered when calibrating the wave spectrum, as well as the magnitude of the wave energy density.

The aim of this study is to calibrate the offshore wave spectrum using the so-called 'shape-focus method'. In this study, offshore wave spectra from global or regional wave models were calibrated against measurements with the shape-focus method. The calibrated wave spectra were then used to drive the third-generation spectrum wave model, SWAN, for nearshore wave transformation. Measured wave spectra were employed to validate the modelled wave data at nearshore locations, to study the impact of offshore wave spectrum calibration and verify the effectiveness of spectrum calibration with the shape-focus method.

\section{METHODOLOGY}

The shape-focus method is to calibrate the wave energy magnitude and take account of spectrum evolution and shape changes. Spectrum calibration with the shape-focus method consists of five steps.

1. Identify key parameters in wave climate and define classifying bins;

Identification of key parameters in wave climate is necessary as wave systems vary at different locations. e.g. swell wave is negligible inside Arabian Gulf, while swell wave is significant in west Africa or US coasts.

2. Group time series of key wave parameters into classifying bins;

This step ensures similar physical processes exist in the same classifying bin, and ultimately helps extend spectrum calibration to the whole hindcast model data in step 5. Figure 2 shows that peaks in the bin of $5.0 \mathrm{~m}<\mathrm{H}_{\mathrm{s}}<5.5 \mathrm{~m}$ are from the southwest, except storm peaks in September. This finding indicates that the mean wave direction, Mdir, is also a key parameter for classification.
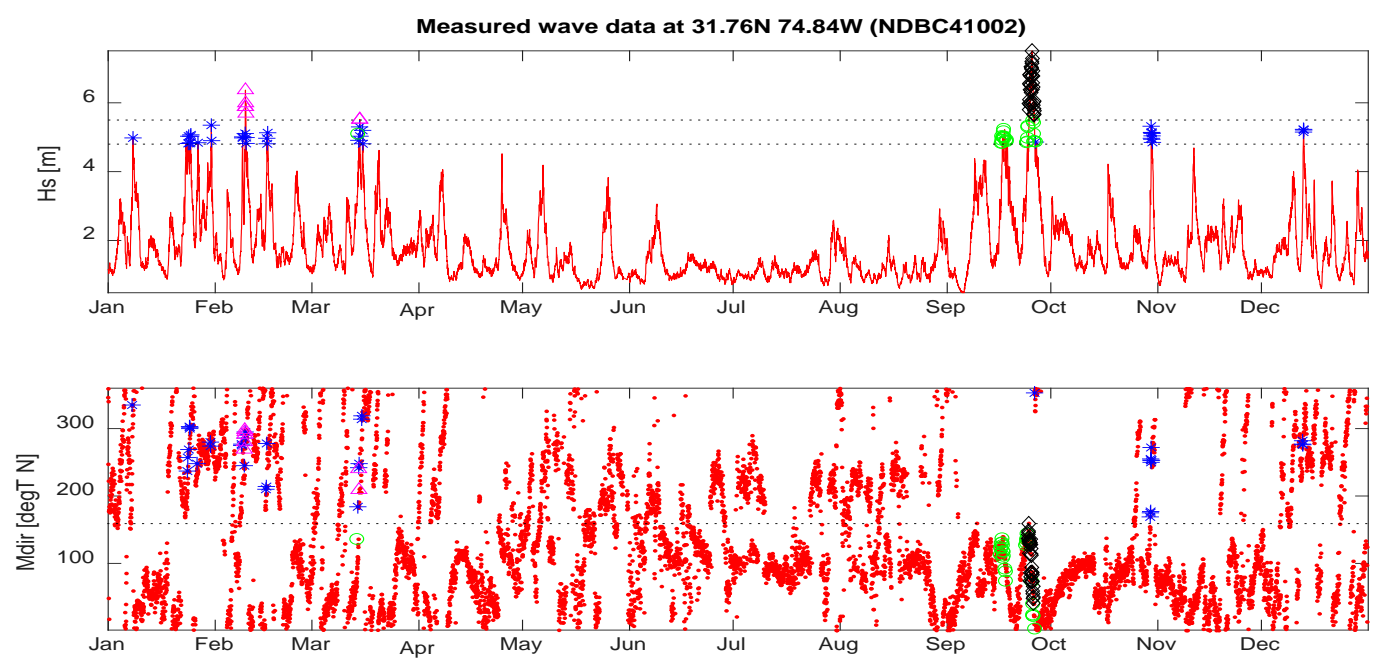

Figure 2 An example of grouping time series of key wave parameters into classifying bins 
3. Form ensembles of instantaneous spectra for each classifying bin;

This step helps analyse spectrum magnitude and shape from a statistical point of view and verify the effectiveness of the classifying bins. Figure 3 presents the ensemble spectra in each classifying bin. It is observed that the classifying bins with Mdir $>160$ degree were not appropriate as spectrum ensembles present a combination of wind sea and swell distributions.
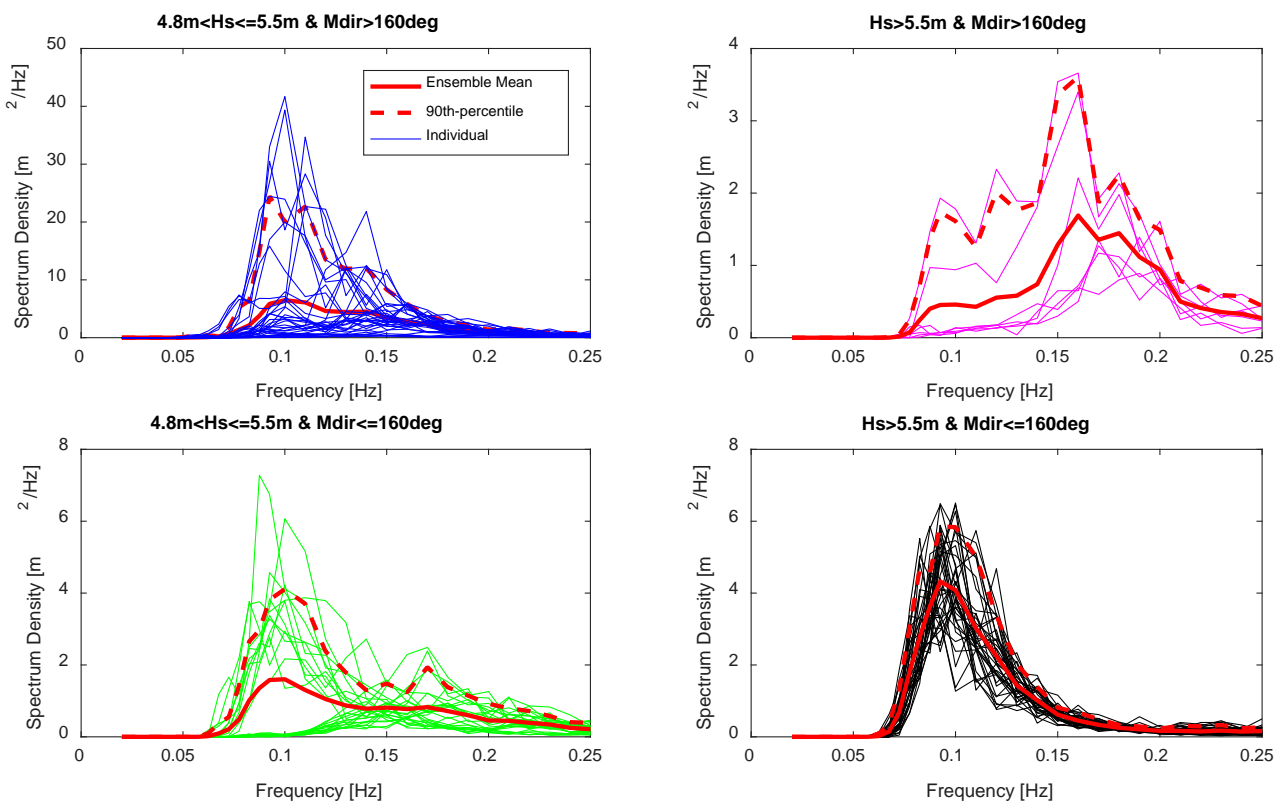

Figure 3 An example of classification and ensembles of wave spectra measured at $31.76 \mathrm{~N} 74.84 \mathrm{~W}$ (WMO41002).

4. Derive calibration factors in each frequency bin using the least square error method;

This step generates the representative calibration factor in each frequency bin and classifying bin (Figure 4). Investigations into other methods, including $95^{\text {th }}$-percentile and ensemble mean, to derive the calibration factor have been carried out, but none of them could represent deviations well for measured and model data. In this study, the calibration factor was set to 1 if the energy density was less than $1 \mathrm{e}-6 \mathrm{~m}^{2} / \mathrm{Hz}$, or the energy density at certain frequencies was smaller than $1 / 10$ the maximum energy density over all frequencies.



Figure 4 An example of calibration factors in each frequency bin for the classifying bin of $1.5 \mathrm{~m}<\mathrm{H}_{\mathrm{s}}<2.0 \mathrm{~m}$ and Mdir $<90^{\circ}$ or Mdir $>180^{\circ}$.

5. Calibrate hindcast spectrum using calibration factors in each frequency bin and classifying bin; Measurements generally cover limited periods, while hindcast model data could last more than 10 years. By categorizing the hindcast model data into classifying bins, this step can calibrate all hindcast spectral by applying calibration factors in each frequency bin derived from step 4. 


\section{MEASUREMENTS}

In this study, measured wave spectra were used to derive the calibration factors for the modelled wave spectra, using the newly proposed shape-focus method.

The measured 2-D spectra were sourced from the National Data Buoy Centre (NDBC, http://www.ndbc.noaa.gov/) to validate this newly proposed method. Two measurement sites were selected, one on the west coast of the USA, representing a single peak wave spectrum, and the other on the east coast of the USA, representing a double peak wave spectrum. The selection of these two sites considered data locations and data availability, wave system and alignment with available model grid points. Table 1 and Figure 5 present an overview of the selected two measurement sites and associated measurement stations.

\begin{tabular}{|c|c|c|c|}
\hline Station No. & Station Name & Location & Site No. \\
\hline 46089 (LLNR 689) & TILLAMOOK & $45^{\circ} 55^{\prime} 29^{\prime \prime}$ N $125^{\circ} 46^{\prime} 17^{\prime \prime} \mathrm{W}$ & 1 \\
\hline 46029 (LLNR 688) & COLUMBIA RIVER BAR & $46^{\circ} 08^{\prime} 36^{\prime \prime} \mathrm{N} 124^{\circ} 29^{\prime} 06^{\prime \prime} \mathrm{W}$ & 1 \\
\hline 44066 & Texas Tower & $39^{\circ} 34^{\prime} 06^{\prime \prime} \mathrm{N} 072^{\circ} 35^{\prime} 08^{\prime \prime} \mathrm{W}$ & 2 \\
\hline 44017 (LLNR 665) & MONTAUK POINT & $40^{\circ} 41^{\prime} 34^{\prime \prime} \mathrm{N} 072^{\circ} 02^{\prime} 56^{\prime \prime} \mathrm{W}$ & 2 \\
\hline 44025 (LLNR 830) & LONG ISLAND & $40^{\circ} 15^{\prime} 03^{\prime \prime} \mathrm{N} 073^{\circ} 09^{\prime} 52^{\prime \prime} \mathrm{W}$ & 2 \\
\hline
\end{tabular}

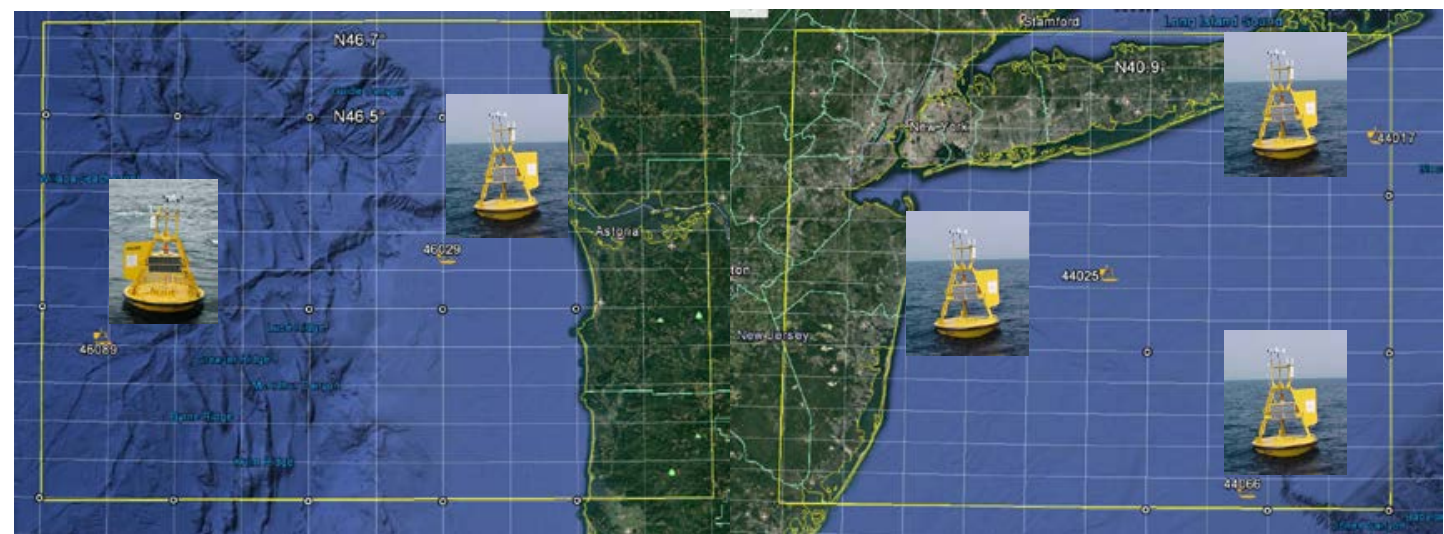

Figure 5 Overview of selected measurements from NDBC. (left) site 1, west coast of the USA and (right) site 2, east coast of the USA

\section{SITE-SPECIFIC WAVE MODELLING}

Since processes of wave generation, dissipation and nonlinear interaction are complex, the SWAN (Simulating Waves Nearshore) wave model was employed to transform offshore wave energy to nearshore regions. The nearshore wave data were then validated with measurements to verify whether spectrum calibration with the shape-focus method improved the accuracy of nearshore waves.

Site-specific wave models were developed for this study to investigate nearshore wave transformation using SWAN. SWAN is a third-generation numerical wave model that may be used to obtain realistic estimates of wave parameters in coastal areas, lakes and estuaries from given wind, bottom and current conditions. The model is based on the wave action balance equation (or energy balance in the absence of currents) with sources and sinks.

2-D spectra sourced from the Fugro Global Wave Database (GWD) were applied to offshore boundaries of the SWAN wave model. The Fugro Global Wave Database was created using a WAVEWATCH III (version 4.18) wave model suite and has been calibrated against available satellite and in-situ buoy data. This wave model is available at a spatial resolution of 0.5 degree and makes use of bathymetry data extracted from the ETOPO1 database. Spatial and temporal-varying meteorological data, consisting of 10-minute averaged wind speed at 10m above MSL, air-sea temperature differences and ice coverage, are sourced from the American National Centers for Environmental Prediction (NCEP) Climate Forecast System Reanalysis (1979-2010) and the Climate Forecast System Version 2 (CFSV2) operational model (2011-2016).

Temporal and spatially varying wind forcing were sourced from the American National Centers for Environmental Prediction (NCEP) CFSR/CFSV2 hindcast model. Climate Forecast System Reanalysis (CFSR) hindcast data are available at 0.312-degree grid resolution and cover the period from 1979 to 2010 in 6-hourly intervals. From January 2011 CFSR has been extended by NCEP's Climate Forecast System Version 2 (CFSV2) operational model. Until the operational CFS is changed, the data produced 
by CFSV2 can be considered as a seamless extension to CFSR. CFSR was designed and executed as a global, high resolution, coupled atmosphere-ocean-land surface-sea ice system to provide the best estimate of the state of these coupled domains over this period.

\section{VALIDATION OF THE SHAPE-FOCUS METHOD}

The nearshore wave data from the site-specific wave modelling were processed and validated against measurements. To show the improvement on wave spectrum shape, the mean wave period, $\mathrm{T}_{\mathrm{m} 01}$, and zero-crossing wave period, $\mathrm{T}_{\mathrm{m} 02}$, were employed to represent the wave spectrum shape during comparisons.

\section{Site 1: West Coast of the USA}

The site 1 is located offshore Oregon, on the west coast of the USA. Winds and waves in this area prevail in the directional sectors of southwest to northwest (Figure 6). The waves from directional sectors of southwest to northwest are a mix of wind sea waves and pacific swell waves, and directions of the wind sea waves align with those of the swell waves. Figure 6 also shows the comparison of wave data between GWD model data and measurements at the offshore point. The significant wave height was overestimated, while mean period and zero-crossing period for large waves were slightly underestimated.
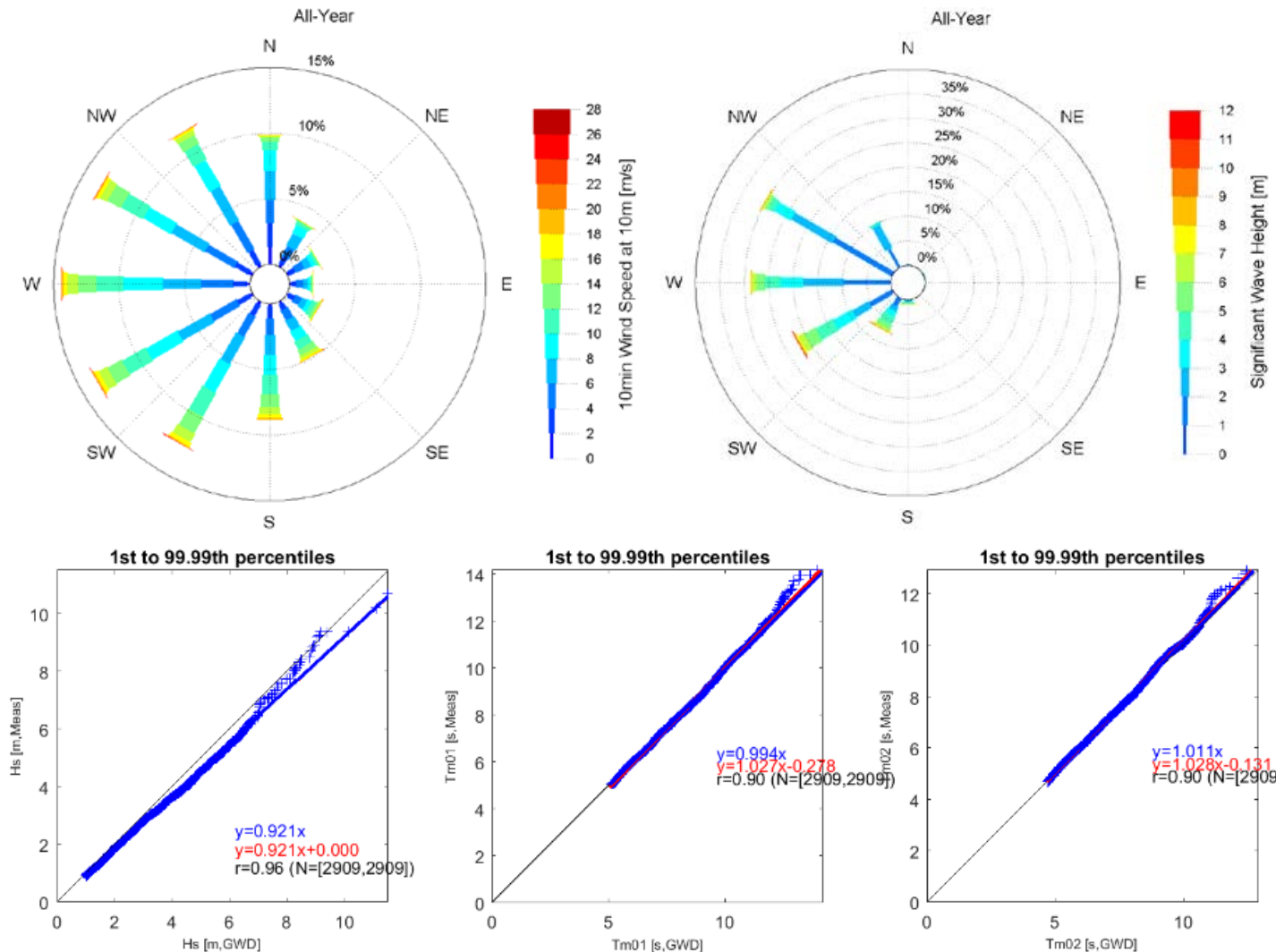

Figure 6 Wind and wave climate at the offshore point in the site 1. (top left) wind rose plot, (top right) wave rose plot, (bottom) Quantile-Quantile plots of wave data between GWD model data and measurements. 



Figure 7 Examples of calibration factor derivation. (left) $1.0 \mathrm{~m}<\mathrm{H}_{\mathrm{s}}<1.5 \mathrm{~m}$, (middle) $4.5 \mathrm{~m}<\mathrm{H}_{\mathrm{s}}<5.0 \mathrm{~m}$, (right) $8.0 \mathrm{~m}<\mathrm{H}_{\mathrm{s}}<8.5 \mathrm{~m}$
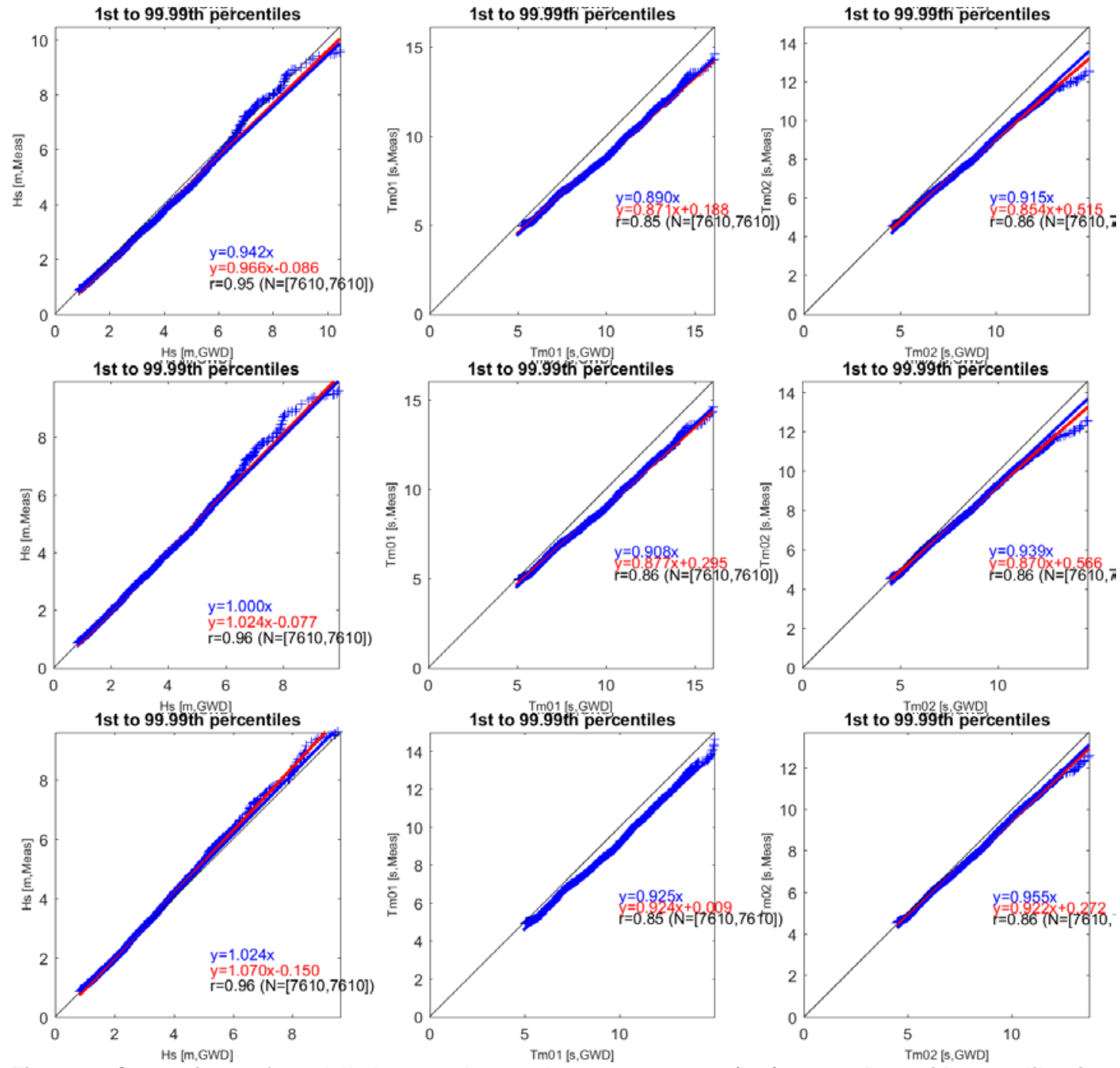

Figure 8 Comparison of modelled wave data and measurements. (top) wave data without calibration, (middle) wave data with constant factoring calibration, (bottom) wave data with shape-focus calibration, on offshore boundary conditions. 
Since waves are mainly from the west, the impact of mean wave direction on classification would be negligible. Therefore, only the wave parameter, Hs, was considered to group wave data into multiple bins with $0.5 \mathrm{~m}$ interval. Figure 7 presents the calibration processes for three representative classifying bins: small Hs with large occurrences $\left(1.0 \mathrm{~m}<\mathrm{H}_{\mathrm{s}}<1.5 \mathrm{~m}\right)$, intermediate $\mathrm{H}_{\mathrm{s}}$ with considerable occurrences $\left(4.5 \mathrm{~m}<\mathrm{H}_{\mathrm{s}}<5.0 \mathrm{~m}\right)$, large $\mathrm{H}_{\mathrm{s}}$ with small occurrences $\left(8.0 \mathrm{~m}<\mathrm{H}_{\mathrm{s}}<8.5 \mathrm{~m}\right)$. Next, instantaneous wave spectra were grouped into ensembles in each classifying bin, for both measurements and offshore model data. Finally, the calibration factor was derived in each frequency bin using the least square error method on differences between modelled data and measurements. The calibration factors are displayed as green dots in Figure 7. It is observed that the calibration factor varies along the frequency bins and accounts for the spectrum shape differences between model data and measurements.

At site 1, since the offshore waves mainly come from the west, using the 2-D spectrum next to the station 46089 can represent the offshore boundary conditions along the whole boundary. Figure 8 presents comparisons of measurements and modelled wave data at nearshore locations. Three scenarios were studied:

- offshore spectrum not calibrated;

- offshore spectrum calibrated with constant factoring method (c.f. Introduction);

- offshore spectrum calibrated with the shape-focus method.

For the case without wave spectrum calibration, the nearshore wave model overestimates the significant wave height, and the quantile distribution of model data is different from that of measurements for peak waves. The wave model also significantly overestimates the mean wave period and zero-crossing wave period. For the case with constant factoring calibration, the model agrees well with measurements in terms of magnitudes of significant wave height, but the quantile distribution is still different for large peak waves. The wave model still significantly overestimates the mean wave period and zero-crossing wave period. In contrast, for the calibration with the shape-focus method, the model performs well for both magnitude and quantile distribution of significant wave height. Furthermore, the model reproduces the mean wave period and zero-crossing wave period well, and the overestimations that existed in other two cases were significantly reduced. Those positive improvements indicate that calibration with the shape-focus method is successful, by not only correcting the magnitude of wave energy, but also adjusting the distribution of wave spectrum energy.

\section{Site 2: East Coast of the USA}

The site 2 is located offshore New York, on the east coast of the USA. In this area the prevailing wind directional sectors are the southwest to the northeast, while waves are distributed in all directional sectors, with large occurrences in the directional sectors from the east to the south (Figure 9). The waves from directional sectors from the east to the south are mainly related to the offshore swell waves, as they come from directions different from the local wind data, which generate local wind sea waves. Figure 9 also shows the comparison of wave data between GWD model data and measurements at an offshore location. The modelled significant wave height was overestimated, while modelled mean wave period and zero-crossing wave period were underestimated. 

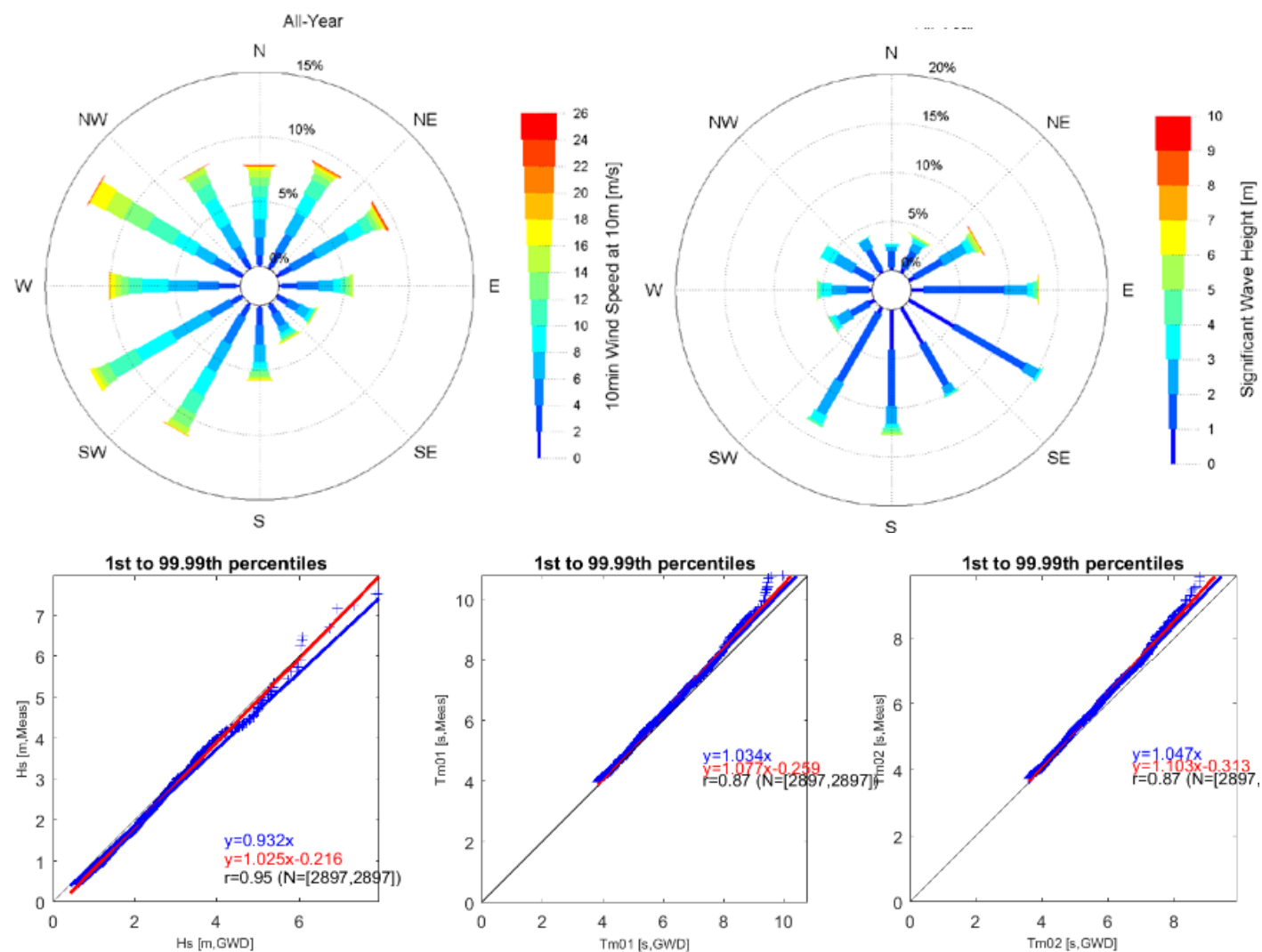

Figure 9 Wind and wave climate at the offshore point in the site 2. (top left) wind rose plot, (top right) wave rose plot, (bottom) Quantile-Quantile plots of wave data between GWD model data and measurements.

Since the wave climate at site 2 presents the mixed wind sea and swell system with direction misalignments, thus, the classification of wave climate must consider both significant wave height and mean wave direction. Two directional bins, $90^{\circ}<=\operatorname{Mdir}<=180^{\circ}$ and Mdir $>180^{\circ}$ or Mdir $<90^{\circ}$, were designed to class time series of wave spectra. Significant wave height, $\mathrm{H}_{\mathrm{s}}$, was also classified into bins with $0.5 \mathrm{~m}$ interval.

Figure 10 presents calibration processes for four representative bins: operational wave heights and in the directional sector of east to south, operational wave height and in the directional sector of southwest to northeast, storm wave heights and in the directional sector of east to south and storm wave height and in the directional sector of southwest to northeast. Next, instantaneous wave spectra, associated with significant wave height and mean wave direction bins, were formed into ensembles, for both the measurements and the offshore model hindcast. Finally, the calibration factor was derived in each frequency bin using the least square error method on the differences between modelled data and measurements. The calibration factors are displayed as green dots in Figure 10. Like the conclusions for site 1 , at site 2 the calibration factor also varies along the frequency bins and accounts for the spectrum shape differences between model data and measurements. 

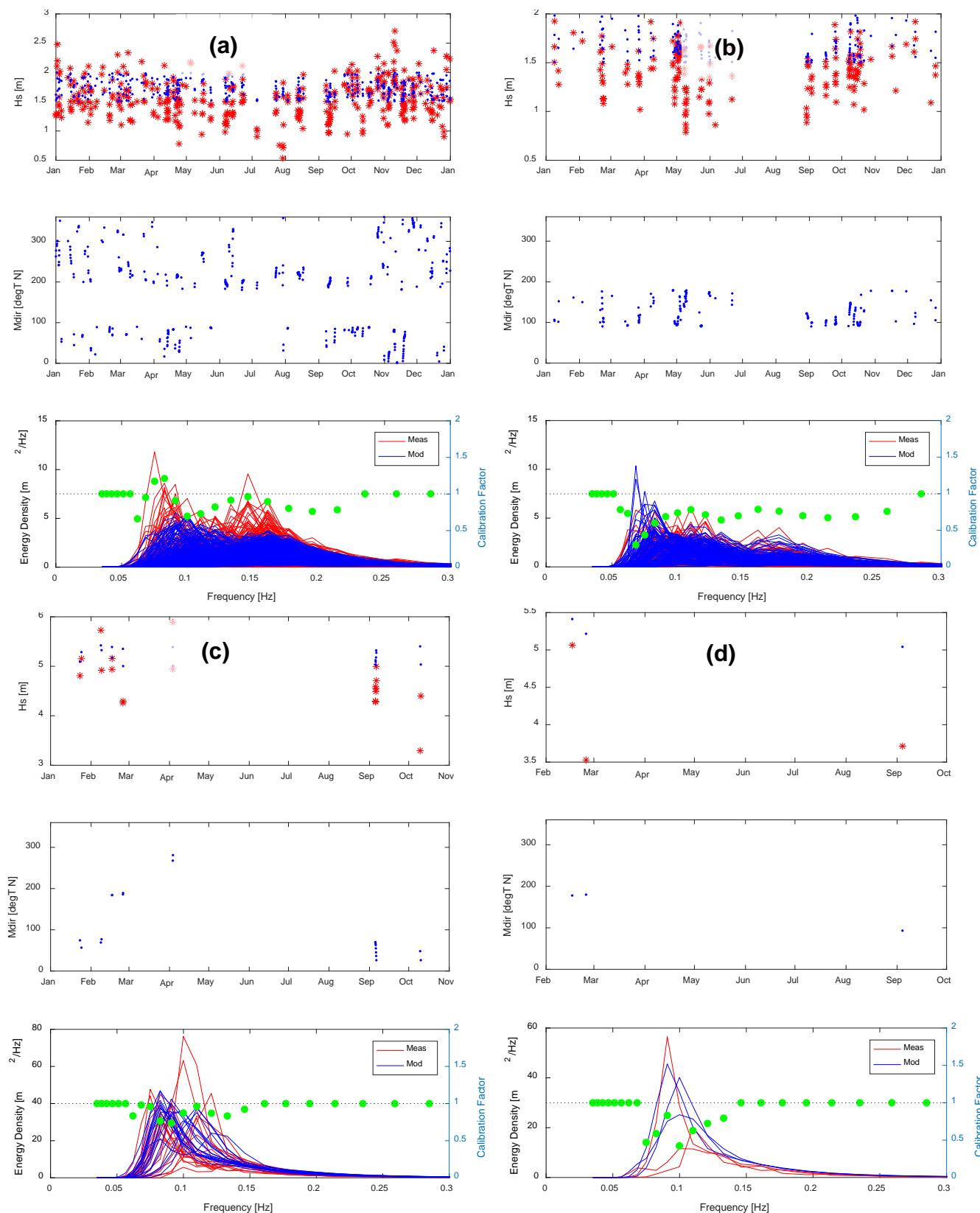

Figure 10 Examples of calibration factor derivation. (a) $1.5 \mathrm{~m}<\mathrm{H}_{\mathrm{s}}<2.0 \mathrm{~m}$ and $\mathrm{Mdir}<90^{\circ}$ or $\mathrm{Mdir}>180^{\circ}$, (b) $1.5 \mathrm{~m}<\mathrm{H}_{\mathrm{s}}<2.0 \mathrm{~m}$ and $90^{\circ}<=\mathrm{Mdir}<=180^{\circ}$, (c) $5.0 \mathrm{~m}<\mathrm{H}_{\mathrm{s}}<5.5 \mathrm{~m}$ and Mdir $<90^{\circ}$ or Mdir $>180^{\circ}$, (d) $5.0 \mathrm{~m}<\mathrm{H}_{\mathrm{s}}<5.5 \mathrm{~m}$ and $90^{\circ}<=$ Mdir $<=180^{\circ}$.

At site 2, offshore waves come from directional sectors of northeast to the southwest, hence the SWAN wave model employs the 2-D spectrum next to the station 44017 for the east boundary condition and 2-D spectrum next to the station 44066 for the south boundary condition (c.f. Figure 5). Figure 11 presents a comparison of measurements and modelled wave data at a nearshore location. For the case without spectrum calibration, the nearshore wave model underestimates the significant wave height, but overestimates the mean wave period. For the case with constant factor calibration, the model only slightly reduced the underestimation of significant wave height and overestimation of mean wave period. However, for the calibration with the shape-focus method, the model improves the accuracy of significant wave height and mean wave period. Wave model data with three scenarios have comparable accuracy for zero-crossing wave period. Those positive improvements in site 2 demonstrate the advantage of spectrum calibration with the shape-focus method. 

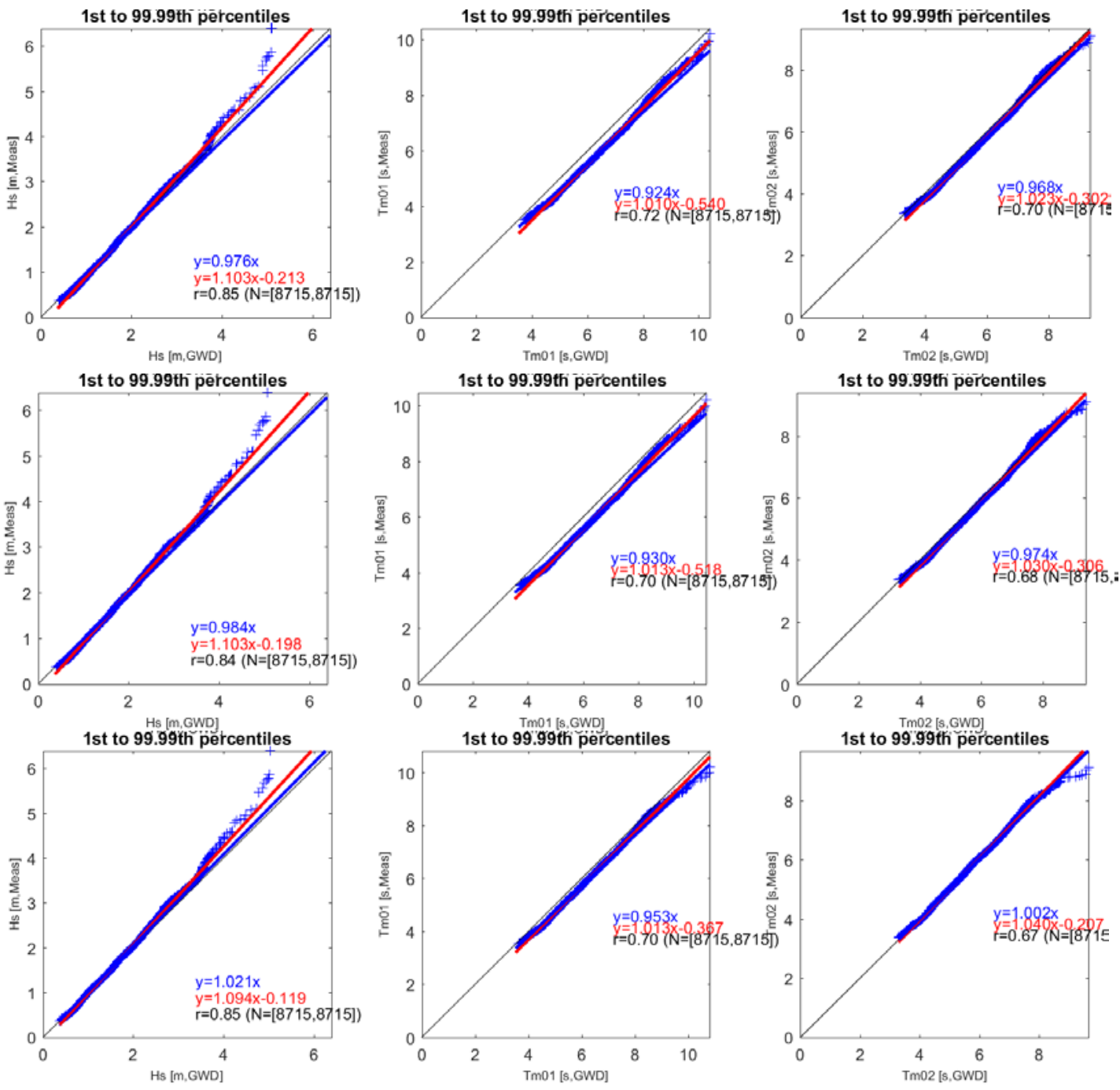

Figure 11 Comparison of modelled wave data and measurements. (top) wave data without calibration, (middle) wave data with constant factoring calibration, (bottom) wave data with shape-focus calibration.

\section{CONCLUSIONS AND FUTURE WORK}

The 'shape-focus' method was proposed for wave spectrum calibration, by deriving calibration factors at each frequency bin from model data and measurements using the least square error approach. The modelled wave spectrum was calibrated with the shape-focus method and then applied to a SWAN wave model to simulate nearshore wave transformation. Nearshore wave spectra were compared with measurements for three scenarios including no calibration, calibration with constant factoring and the shape-focus method. Results show that calibration with shape-focus method improves wave data accuracy significantly for both significant wave height and mean wave periods. The shape-focus method thus provides a more accurate solution to calibrating wave spectra for nearshore wave modelling.

In this study, the calibration with the shape-focus method was applied to the frequency spectrum only. Since the energy in directional domain affects the wave refraction and wave spreading, the directional spectrum shape might also be of importance, particularly for mis-aligned wind sea and swell systems. Therefore, applications of the shape-focus method shall be extended to 2-D directional spectra in the future.

\section{REFERENCES}

Tucker, M.J. (1991): Waves in Ocean Engineering. Measurement, analysis, interpretation. Ellis Horwood. New York, London, pp, 431.

Ardhuin, F., T.H. Herbers, K.P. Watts, G.P. van Vledder, R. Jensen, and H.C. Graber (2007): Swell and Slanting-Fetch Effects on Wind Wave Growth. J. Phys. Oceanogr., 37, pp, 908-931, https://doi.org/10.1175/JPO3039.1 